\title{
EL PORTAFOLIO COMO ESTRATEGIA DE EVALUACIÓN EN CARRERAS DE POSGRADO INTER Y TRANSDISCIPLINARIAS ${ }^{1}$
}

\begin{abstract}
Marta Fracapani* y Marisa Fazio**
Resumen: La Maestría en Bioética de la Universidad Nacional de Cuyo reúne profesionales de ciencias sociales y humanas y de ciencias duras, y acentúa especialmente la formación de actitudes como la tolerancia, la apertura al diálogo, el juicio crítico, entre otros. El objetivo del estudio que describe este artículo es evaluar si el portafolio puede ser considerado como una herramienta auténtica de evaluación de los aprendizajes en un posgrado en el cual el perfil que se espera formar está constituido en su mayor parte por actitudes.

Analizadas dos cohortes, los resultados parciales demuestran que permite evaluar actitudes pero que presenta dificultades al momento de involucrar a los alumnos en dicho tipo de evaluación y en establecer una valoración lo más objetiva posible. Actualmente, resta sólo una cohorte para finalizar la investigación.
\end{abstract}

Palabras clave: evaluación de los aprendizajes, formación de posgrado, carreras inter y transdisciplinarias en el área salud

\section{THE BRIEFCASE AS AN EVALUATION STRATEGY FOR POST, INTER AND TRANSDISCIPLINARY STUDIES}

\begin{abstract}
The Bioethics Magister offered by de National University of Cuyo gets together social and human sciences professionals and those of the hard sciences, and, among others, insists especially on creating attitudes, such as tolerance, willingness for dialogue and critical judgement. The studies' goal that this work describes consists in evaluating if the portfolio may be considered as an authentic tool to evaluate the apprenticeship of a postgraduate study, where as its most expected profile consists mainly on forming attitudes.

Once two cohorts have been analysed, the partial results demonstrate that it is possible to evaluate attitudes, but that it presents difficulties when needing to involve students in that type of evaluation and in establishing an appraisal as objective as possible. At the time being only one cohort is still missing to put an end to the investigation
\end{abstract}

Key words: apprenticeships' evaluation, forming health-care post, inter and transdisciplinary careers

\section{O PORTFOLIO COMO ESTRATÉGIA DE AVALIAÇÃO NOS CURSOS DE PÓS-GRADUAÇÃO INTER E TRANS-DISCIPLINAR}

Resumo: O mestrado em Bioética da Universidade Nacional de Cuyo, reune profissionais da área de ciëncias sociais, ciências humanas e ciências duras e acentua especialmente a formação de atitudes tais como a tolerânica, abertura ao diálogo e juízo crítico entre outros valores. O objetivo deste estudo relatado neste artigo é avaliar se o portfolio pode ser considerado como uma ferramenta autêntica de avaliação da aprendizagem na pós-graduação, na qual o perfil que se espera formar é constituido preponderantemente por atitudes.

Após análise de dois cortes, os resultados parciais demonstram que permitem avaliar as atitudes, porém apresenta dificuldades no momento de envolver os alunos neste processo de avaliação de aprendizagem, bem como no estabelecimento de uma valoração o mais objetiva possível. Atualmente permanece ainda um corte para a finalização da pesquisa.

Palavras chave: avaliação da aprendizagem, formação em pós-graduação, cursos inter e transdisciplinares na área da saúde

1 Estudio de tres cohortes de la Maestría en Bioética, Facultad de Ciencias Médicas, Universidad Nacional de Cuyo y Programa de Bioética OPS/ OMS.

* Doctora en Medicina, Universidad Nacional de Cuyo. Maestría en Bioética, Facultad de Ciencias Médicas, Argentina

** Magíster en Evaluación Educacional, Universidad Nacional de Cuyo, Facultad de Filosofía y Letras, Argentina

Correspondencia: mfracapani@millic.com 


\section{Introducción}

La evaluación de los aprendizajes en la formación de posgrado es un gran desafío, especialmente en carreras inter y transdisciplinarias. Actualmente, instituciones educativas de posgrado, abocadas tanto a ciencias sociales como a ciencias duras, consideran que la evaluación más objetiva es la que permiten los instrumentos estructurados y semiestructurados del enfoque curricular técnico.

El presente estudio tiene como propósito medir si el portafolio puede ser considerado una herramienta auténtica de evaluación de los aprendizajes en la Maestría en Bioética de la Universidad Nacional de Cuyo, posgrado que apunta a la formación de un perfil profesional en el cual la mayor parte de las competencias son actitudinales: tolerancia, apertura, diálogo, juicio crítico, responsabilidad, respeto, por ejemplo. A su vez, dichas actitudes atraviesan transversalmente todas las competencias conceptuales y procedimentales propuestas en el plan de formación.

Partiendo de las características del perfil profesional, los responsables de la formación consideraron que no era significativo adoptar un sistema de evaluación de los aprendizajes sólo desde el enfoque técnico. Con esto nos referimos a la aplicación exclusiva de instrumentos tales como opción múltiple, cuestionarios de preguntas cerradas, pruebas de completación, entre otros, en los cuales se invita al maestrando sólo a reproducir los conocimientos brindados en cada uno de los módulos de la carrera. Así, se agregaron evaluaciones "abiertas" y cualitativas, incorporándose la elaboración de ensayos, los grupos de discusión y los trabajos de producción que permitieran demostrar la creatividad en la resolución de problemas o aportes innovadores como resultado de actitudes que deben estar presentes en todo bioeticista.

No obstante, al momento de emitir un juicio de valor lo más objetivo posible sobre el aprendizaje del maestrando, se seguía evaluando en forma estancada por módulos. Esta modalidad no permitía aún valorar el aprendizaje integral del alumno ni el grado de desarrollo de las principales competencias actitudinales y transversales que componen el perfil. Se decidió entonces utilizar el portafolio (aplicado a dos cohortes y que en 2007 y 2008 se utilizará con la tercera) para obtener los resultados finales de la presente investigación.
Para el desarrollo del proyecto se analizó bibliografía referida al portafolio como instrumento de evaluación formativa, teniendo en cuenta, en primer lugar, el estado actual de la evaluación de las actitudes. Para ello, usamos algunas reflexiones de Pedro Ahumada, que consideramos refleja en forma acabada el tema objeto de estudio de la presente investigación(1).

Respecto de la evaluación de las actitudes, las autoras reconocen que los procedimientos usados hasta hoy para evaluar estas competencias han estado fuertemente limitados por el supuesto de que éstas se presentan en un mismo momento y se desarrollan por igual en todos los componentes de un grupo-curso. Esta falacia ha conducido a que se sigan utilizando procedimientos tipo encuesta y pautas de observación rígidas que suponen reacciones semejantes de todos los sujetos frente a un determinado estímulo.

Las escalas Lickert y Thurstone y el diferencial semántico de Osgood permitieron estructurar la mayoría de los instrumentos de observación de comportamientos actitudinales y cuya información no siempre finaliza en una interpretación acertada o, por lo menos, reconocida como cierta por el sujeto evaluado o por quienes lo conocen.

El problema surge cuando la intención es evaluar formalmente, intentando hacer públicas las estimaciones y procurando certificar un nivel de "calidad" actitudinal de los estudiantes. En la práctica evaluativa se mezclan una dimensión relacionada con el problema metodológico, asociada directamente con las técnicas e instrumentos que se emplean, con otra que manifiesta el problema ético, es decir, con qué grado de legitimidad se pueden evaluar las actitudes de una persona. La dimensión técnico-metodológica, sostienen las autoras, debe estar siempre subordinada a la ética-moral.

Se comienzan a plantear entonces ideas contradictorias sobre lo que debe o no ser una educación en actitudes, y sobre la posible neutralidad que deberían mantener los evaluadores; por ejemplo, acerca de la subjetividadobjetividad de la evaluación, que afecta profundamente este intento evaluativo, y sobre la validez de los procedimientos que se adoptarían para llevarlo a cabo.

Ahumada propone un marco orientador en la formación de actitudes que, a la vez, permite enfocar adecuadamente la evaluación. La enseñanza y la evaluación crítica de actitudes implica considerar "la articulación entre actitudes individuales y los modos de pensar que 
caracterizan a las comunidades en que viven los estudiantes". Como propuesta pedagógica, exige que los alumnos puedan conectar sus personales afirmaciones de valores y actitudes con un marco más amplio de significados en que éstos puedan tener sentido.

En consecuencia, evaluar las actitudes de los estudiantes no puede ser un objetivo en sí mismo, que convierta a la actividad de clase en una acción instrumental para conseguir determinados objetivos (entre ellos los actitudinales). Como han señalado Peters y Stenhouse, "son las cualidades, los valores y los principios intrínsecos al propio proceso educativo, y no los resultados objetivos extrínsecos que se consiguen, los que hacen educativa una actividad".

Las actitudes son contenidos siempre presentes en el aprendizaje de cualquier tipo, es decir, el profesor, cada vez que trata determinados contenidos, puede y debe hacer referencia a los valores permanentes, a normas sociales vigentes o estimular una reacción positiva hacia los contenidos que presenta. Por consiguiente, la toma de posición de un alumno frente a cada tema señala un acercamiento actitudinal que, si bien resulta difícil precisarlo específicamente, puede ser apreciado en general como una respuesta favorable del estudiante hacia valores de mayor trascendencia. En este modelo se considera la educación ética y moral primordialmente como autónoma, es decir, los valores, normas y actitudes son construidos por el propio sujeto.

El desarrollo actitudinal se entiende como un proceso lento de internalización y, por tanto, su evaluación debe ser asumida como una recolección de evidencias a mediano y largo plazo. Desde el momento en que el estudiante "recibe" el estímulo hasta que "responde" transcurren tiempos variables que hacen imposible una evaluación uniforme. La presente investigación tiene como hipótesis que el portafolio es un instrumento significativo para la evaluación "auténtica" de actitudes. Ha sido considerado como una alternativa a los sistemas tradicionales de evaluación por "pruebas" $(2,3)$, porque recoge las evidencias del aprendizaje en el momento en que el alumno se está aproximando a los conocimientos, y como un medio facilitador de la interactividad entre profesor y alumno en el más genuino proceso dialógico de enseńar y aprender.

Ahumada lo define como "una forma alternativa de evaluación que comprende la compilación sistemática del trabajo del estudiante, con el propósito de evaluar el progreso académico durante un período de tiempo determinado". Es un conjunto particular de actividades y trabajos que recoge las producciones de cada estudiante, tanto las que evidencian lo que ha aprendido como aquellas que se consideran sólo aproximaciones. Esta colección se materializa en una carpeta individual con distintas secciones (clasificaciones), establecidas en función de los propósitos del curso, asignatura o unidad en forma concertada con el profesor(4).

Una ventaja es la posibilidad que tiene el estudiante de decidir qué productos colocar en el portafolio, cómo describir lo que este producto representa y relacionarlo de manera dinámica con el conocimiento. Además, sus producciones pueden ser usadas para motivar discusiones entre los propios estudiantes o con el profesor $(5,6)$.

Por medio de los trabajos realizados, estudiantes y profesores pueden establecer interacciones sobre cómo se alcanza el conocimiento y cómo se construye el aprendizaje. También, los profesores pueden obtener mucha información analizando los productos que los alumnos eligen poner en sus portafolios, en particular, analizando los diferentes sentidos que el conocimiento adquiere para éstos a lo largo del tiempo(7).

Una característica importante es que deben ser construidos íntegramente por el estudiante y su evaluación parcial y final negociadas entre él y el profesor, de modo que le permita tener certeza que está "construyendo" un camino válido hacia su conocimiento(8). Requiere de una cuidadosa planificación por parte del docente; supone intencionalidades pedagógicas y opciones metodológicas dirigidas a favorecer determinadas estrategias de aprendizaje en los alumnos. Asimismo, requiere un clima apropiado entre docentes y alumnos para las interacciones que esta técnica supone.

El punto de partida para esta experiencia lo constituye la unidad de aprendizaje, reconocida como un conjunto articulado de elementos que, posteriormente, admite ser evaluada separadamente como proceso y globalmente como producto. Hacia este propósito intencionado, formulado o no explícitamente, concurren los alumnos con su bagaje de conocimientos previos y con la mediación de un docente que facilita su logro mediante actividades seleccionadas por él o propuestas por los propios estudiantes, cuya realización les permite dominar determinados "procesos" o materializarlos 
en un "resultado o producto final global" que tenga sentido y significado para el estudiante.

Si concebimos el aprendizaje como una experiencia global, no es posible considerar este proceso como algo lineal, destinado a alcanzar un solo propósito u objetivo; cada alumno puede darle a cada actividad un significado más amplio y, por lo tanto, diferente al que ha fijado su profesor. Esta técnica es útil para evaluar contenidos conceptuales, procedimentales y actitudinales. Con el fin de garantizar su validez y confiabilidad, es conveniente considerar una serie de criterios que permitan seleccionar los elementos susceptibles de ser incorporados. Sería un contrasentido que el profesor sólo se fijara en las formas o en la cantidad de elementos que contiene el portafolio. Por ello, es importante que se involucre en el proceso evaluador, dando a conocer su parecer por escrito como principal mediador entre los contenidos disciplinares y el aprendizaje de sus alumnos(9).

Teniendo en cuenta lo expuesto en el marco de referencia, son objetivos de este trabajo de investigación:

- Determinar el valor del portafolio como instrumento de evaluación formativa, especialmente de actitudes, en un proceso de formación inter y transdisciplinaria.

- Contribuir a ampliar y complementar la variedad de instrumentos de evaluación de los aprendizajes de actitudes en carreras de posgrado inter y transdisciplinarias.

\section{Hipótesis}

El portafolio es un instrumento significativo de evaluación formativa de las actitudes en carreras de posgrado inter y transdisciplinarias.

\section{Metodología y resultados}

Se enfocó la investigación desde una perspectiva procesal y sistémica. El diseño, implementación y metaevaluación del sistema de portafolio, como principal herramienta de evaluación de los aprendizajes actitudinales y de formación integral durante el desarrollo del Magíster, es un largo proceso de internalización y desarrollo, manifestado en las dos cohortes que lo han utilizado. En ambas se inició la experiencia desde cero; ninguno de los alumnos sabía en qué consistía y sus experiencias evaluativas giraban en torno a instrumentos de tinte cuantitativo, propios del enfoque curricular técnico.
Respecto de la primera cohorte, se les informó qué era un portafolio, en qué consistía y cómo se podía estructurar. Se dio total libertad a los maestrandos, ya que se supuso características de sujetos de aprendizaje de posgrado. La guía para su diseño y desarrollo estuvo a cargo de los directivos y del pedagogo como una primera experiencia. No se involucró a los docentes, ya que sólo estaban con los alumnos durante una semana y perdían contacto directo con ellos.

El resultado de esta primera cohorte fue satisfactorio, si bien se contó sólo con aproximaciones de portafolios. Éstas se constituyeron en "diarios de clase" de algunos de los módulos con los cuales los maestrandos tuvieron mayor afinidad o se sintieron con dificultades. Respecto de la segunda cohorte, nos encontramos con que sólo algunos tenían experiencia en portafolios y el resto estaba en las mismas condiciones que la cohorte anterior. La información sobre qué era un portafolio, en qué consistía y por qué y para qué se utilizaba en la maestría fue más detallada, objeto de una clase. En dicha instancia las indicaciones y la modalidad de trabajo fueron mediante pautas, porque la utilización del portafolio, como un espacio de auto, co y heteroevaluación, es una instancia que debe ir desde los responsables de la formación hacia la autonomía del maestrando. Por tal motivo, se realizó un seguimiento, con momentos para trabajar en forma compartida con un compañero del grupo, elegido por los directivos y el pedagogo según las características y perfil de los sujetos de aprendizaje. La estrategia de que el portafolio fuera en pareja respondía a ejercitar actitudes propias de un bioeticista, en este caso, la apertura al diálogo y la tolerancia, entre otras.

En esta etapa las pautas de confección fueron las siguientes:

- Debía dividirse por seminarios. La Maestría en Bioética está compuesta por siete seminarios.

- Cada seminario, a su vez, debía contar con subapartados: instancias de aprendizaje obligatorias, optativas y aportes personales. Las primeras constituidas por las actividades propuestas por los responsables de la formación; las segundas no formaban parte del currículum obligatorio y apuntaban a potenciar, enriquecer o superar el grado de desarrollo de alguna competencia. Los aportes personales surgían del entusiasmo o interés por algún tema y se compartían con el grupo-clase.

- A su vez, cada uno de los subapartados se dividían 
en: actividades de aprendizaje, preparadas por los docentes bajo la forma de guías, para el primer subapartado, y, para el segundo, instancias de aprendizaje elaboradas por los mismos autores del portafolio para aplicar al grupo. Por semana, cada pareja de portafolio debía preparar una clase con un tema que formaba parte de la unidad de aprendizaje del seminario. La Maestría tiene una duración de dos años, con un cursado intensivo de siete semanas por cada ańo; por semana se desarrolla un seminario. Aparte de las actividades de aprendizaje, se agregaban las de evaluación (auto, co y heteroevaluación) más un tercer apartado de bibliografía aportada por los docentes, por los compañeros y por los mismos autores para enriquecer la ofrecida por la Maestría. El apartado evaluación contenía tanto la medición de los aprendizajes como la que los maestrandos realizan de los procesos de enseñanza, todo bajo la tutoría de guías.

- Por semana, se dedicaba un espacio para la elaboración del portafolio y para compartir con el grupo-clase los avances, logros y dificultades.

De esta segunda cohorte, todos terminaron el portafolio. La diferencia entre ambas cohortes sólo radica en su grado de desarrollo y/o en la profundidad, pero todos cumplieron con los requisitos mínimos.

\section{Discusión}

Ventajas:

- Se pueden identificar las actitudes que trae el alumno a la Maestría y las que va desarrollando con relación al programa de formación y al perfil del biotecista que se pretende formar.

- Permite valorar si el sujeto alcanza o no las competencias principales para ejercer el perfil profesional propuesto.
- El maestrando evidencia en las actividades obligatorias, y más en las optativas y aportes personales, las dificultades que desea superar y las fortalezas por potenciar.

- Las instancias de auto, co y heteroevaluación de los aprendizajes y del proceso de enseńanza lo tornan un verdadero protagonista de su proceso de formación. Desarrolla responsabilidad, creatividad e innovación para hacer propuestas superadoras, tanto de su propio aprendizaje como de las estrategias de enseñanza que transita. El diálogo y la apertura se tornan algo fundamental.

- En cuanto a los trabajos, determinan propósitos y existe coherencia entre éstos y los trabajos realizados. Demuestran objetividad en su auto y coevaluación.

Desventajas:

- Resistencia en un primer momento, debido a la falta de internalización de un instrumento de evaluación formativa de proceso que enfatice el aprendizaje y evaluación, especialmente de actitudes.

- Falta de tiempo y hábitos para sistematizar en un posgrado todas las experiencias de aprendizaje.

- Carencia de hábitos en actitudes como el diálogo, compartir, reconocer el error y no imponer la propia postura.

En los años académicos 2007 y 2008, a partir de las dos experiencias anteriores, se aplicará el portafolio a la tercera cohorte de la Maestría, finalizando así la investigación.

Se invita a los responsables de formación de posgrado para que, teniendo en cuenta la naturaleza de las especialidades que dictan, apliquen el portafolio como una estrategia significativa, no sólo de evaluación sino de un verdadero aprendizaje.

\section{Referencias}

1. Ahumada Acevedo P. La evaluación en una concepción de aprendizaje significativo. Santiago de Chile: Ediciones Universitarias de Valparaíso; 2002: 91-119.

2. Font A. Una experiencia de autoevaluación y evaluación negociada en un contexto de aprendizaje basado en problemas. Revista de la REDU; 3(2): 100-112.

3. Ahumada Acevedo P. Principios y procedimientos de evaluación educacional. Santiago de Chile: Ediciones Universitarias de Valparaíso; 2003.

4. Ahumada Acevedo P. Tópicos de evaluación en educación. Santiago de Chile: Ediciones Universitarias de Valparaíso; 1989.

5. Casanova M. Manual de evaluación educativa. Madrid: Editorial La Muralla. SA; 1999. 
6. Montbrun M, Fracapani M, Fazio M, et al. Desarrollo de un portafolio como herramienta de evaluación formativa en educación médica de grado. Proyecto de Investigación (2002-2004). Mendoza: Universidad Nacional de Cuyo, Secretaría de Ciencia y Técnica; 2004.

7. Klenowxki V. Desarrollo de portafolios para el aprendizaje y la evaluación. Madrid: Narcea; 2004: 45.

8. Sorrell J, Brown H, Silva M, Kohlenberg E. Use of writing portafolios for interdisciplinary assessment of critical thinking outcomes of nursing students. Nursing Forum 1997; 32(4): 12-25.

9. Williams J. The clinical notebook: Using student portafolios to enhance clinical teaching learning. Journal of Nursing Education 2001; 40(3): 135-138.

Recibido: 3 de mayo de 2008 .

Aceptado: 12 de mayo de 2008 . 